\title{
Prevalence of burnout and its relationship to health status and social support in more than 1000 subspecialty anesthesiologists
}

\author{
Steve A Hyman (1) , ${ }^{1}$ Elizabeth Borg Card, ${ }^{2}$ Oscar De Leon-Casasola, ${ }^{3,4}$ \\ Matthew S Shotwell, ${ }^{5}$ Yaping Shi, ${ }^{5}$ Matthew B Weinger ${ }^{6,7}$
}

\begin{abstract}
- Prepublication history and additional material is published online only. To view please visit the journal online (http://dx. doi.org/10.1136/rapm-2020 101520).
\end{abstract}

For numbered affiliations see end of article.

Correspondence to Dr Steve A Hyman,

Anesthesiology, Vanderbilt University Medical Center, Nashville, TN 37232-2102, USA steve.hyman@vumc.org

Received 3 April 2020 Revised 9 January 2021 Accepted 12 January 2021 Published Online First 11 February 2021

\section{Linked}

- http://dx.doi.org/10.1136/ rapm-2021-102530

\section{Check for updates}

(C) American Society of Regional Anesthesia \& Pain Medicine 2021. No commercial re-use See rights and permissions. Published by BMJ.

To cite: Hyman SA, Card EB, De Leon-Casasola 0 , et al. Reg Anesth Pain Med 2021:46:381-387.

\section{ABSTRACT}

Background Physician burnout may be at 'epidemic' proportions due to factors associated with modern healthcare practice and technology. Practice attributes vary appreciably among subspecialists. Understanding burnout incidence and its associated factors could illuminate potential causes and interventions. We evaluated burnFout, mental and physical health, and social support and coping skills in acute and chronic pain physicians and pediatric and cardiac anesthesiologists. Methods We administered the Maslach Burnout Inventory Human Services Survey (MBI-HSS), a two-item self-identified burnout measure, the Veterans RAND 12-item Health Survey and the Social Support and Personal Coping Survey to subspecialty society members practicing acute and chronic pain management, pediatric anesthesiology and cardiac anesthesiology. Multivariable regression analysis compared the groups, and adjusted burnout prevalence was compared with an all-physician and an employed general population sample.

Results Among 1303 participants (response rates $21.6 \%-35.6 \%$ among the subspecialty groups), $43.4 \%$ met established burnout criteria (range 30.0\%-62.3\%). Chronic pain physicians had significantly worse scores (unadjusted) than the other three groups of subspecialty anesthesiologists, the all-physician comparator group and the general population comparator group. Mental health inversely correlated with emotional exhaustion and depersonalization in all groups. Self-identified burnout correlated with the full MBI-HSS $(R=0.54$; positive predictive value of $0.939(0.917,0.955))$. Physicians' scores for personal accomplishment were higher than population norms.

Conclusions This study provides data on burnout prevalence and associated demographic, health and social factors in subspecialist anesthesiologists. Chronic pain anesthesiologists had significantly greater burnout than the other groups. The self-identified burnout metric performed well and may be an attractive alternative to the full MBI-HSS.

\section{INTRODUCTION}

Burnout continues to concern individuals in all professions. First described in child mental health workers, ${ }^{12}$ it is a phenomenon that affects many workers, although the three dimensions-emotional exhaustion, depersonalization and personal accomplishment-have different patterns in different professions. ${ }^{3}$ One version of the 'gold-standard'
Maslach Burnout Inventory, the Maslach Burnout Inventory-Human Services Survey (MBI-HSS) has been extensively validated for use in clinicians. ${ }^{4}$

Burnout prevalence varies among medical specialties, and anesthesiologists report an incidence in the median range compared with medical colleagues in other specialties. ${ }^{3}$ A recent study suggests that up to half of all anesthesiology residents and recent graduates report symptoms of burnout ${ }^{5}$ and $15 \%$ of experienced anesthesiologists show a high risk of burnout. ${ }^{6}$ In parallel, anesthesiologists report low job satisfaction based primarily on diminished job control and degraded work-life balance. ${ }^{6}$

Anesthesiologists who work in the operating room (OR) are more insulated from the 'vicissitudes' of many of the known contributors to burnout experienced in most physician practices. ${ }^{7}$ While OR anesthesiologists interact with patients preoperatively, they usually do not have long-term patient relationships. In contrast, like surgeons or some subspecialty internists, anesthesiologists specializing in chronic pain management typically have a busy office practice and also perform invasive procedures. They have extended patient relationships with chronic or cancer pain patients. Unlike OR anesthesiologists, chronic pain physicians must daily deal with their patients' persistent pain, drug dependence, myriad mental and behavioral health problems, and other psychosocial issues. Although there are limited data on burnout in palliative care physicians, ${ }^{89}$ and none for chronic pain physicians, one might suppose that chronic pain physicians would have a different incidence of burnout than that found in other anesthesiologists and other physicians in general. We were interested in understanding how these subspecialist groups differed in their burnout incidence and whether there were other easily measured attributes of the groups associated with any differences (eg, demographics, mental or physical health). Further, because the MBI-HSS is a proprietary multiquestion survey tool, the study of clinician burnout could be advanced by validating a simple, opensource, univariate measure of burnout. For this, we chose to study concurrently a simple two-item selfreported measure that allows respondents to selfidentify as being or having been burned out.

Thus, three main research questions drove our study design:

1. Is the risk of burnout as measured by the MBIHSS different across the four groups of subspecialty anesthesiologists, those primarily 
practicing chronic pain management, acute pain management, cardiac anesthesiology or pediatric anesthesiology?

2. What is the concordance between results from the MBI-HSS and the simple unidimensional self-reported measure?

3. Does the impact of easily measured factors that have been previously associated with the risk of burnout differ between these four anesthesiologist subspecialty groups?

\section{METHODS}

\section{Sampling and participants}

The Behavioral Sciences Institutional Review Board at Vanderbilt University approved this study. Participants belonged to at least one of three different subspecialty anesthesia societiesthe American Society of Regional Anesthesia and Pain Medicine (ASRA), the Society for Pediatric Anesthesia (SPA) and the Society of Cardiovascular Anesthesiologists (SCA). ASRA member respondents were specifically asked to identify whether they dealt primarily with chronic or acute pain. Chronic pain physicians deal with patients who have had pain for more than 3 months. Acute pain physicians provide both regional and parenteral analgesia in the perioperative period. The ASRA cohort was thus further segmented as possible into those practicing primarily acute versus chronic pain. Recognizing the nomenclature simplification, for clarity henceforth, participants from SPA will be referred to as 'pediatric' while those from SCA will be referred to as 'cardiac'.

Each society office contacted their full membership by email, inviting them to participate. After accepting the initial invitation, participants received a link to the survey housed on Research Electronic Data Capture, a secure database. ${ }^{10}$ Failure to respond was followed by subsequent invitations ${ }^{3} 11$ at approximately 10-day intervals. Data were collected from December 2016 to April 2017. ASRA independently sent additional email reminders and a postcard reminder to its domestic members.

To minimize bias, potential participants were not informed of the specific purpose of the study. To ensure anonymity, each questionnaire was numerically coded, with the code unavailable to either the test administrators or scorers. Participants were free to omit any question or not to complete the survey.

The survey of approximately 60 questions assessed the magnitude of burnout risk, physical health problems, mental health problems, social support and personal coping among respondents. ${ }^{6}$ Participants were presented with the MBI-HSS, ${ }^{4}$ the selfidentified burnout questions, the Social Support and Personal Coping Survey ${ }^{6}$ and the Veterans RAND 12-item Health Survey. ${ }^{12}$ The number of questions varied among participants, as branching logic added or eliminated questions based on the responses.

\section{Survey instruments}

Maslach Burnout Inventory Human Services Survey

The MBI-HSS ${ }^{413}$ is a well-validated tool that has been administered to hundreds of thousands of healthcare providers. MBIHSS consists of 22 questions that evaluate the three dimensions of burnout-emotional exhaustion (nine questions), depersonalization (five questions) and personal accomplishment (eight questions). Subjects use a seven-point Likert scale (encoded 0-6) for their answers. Personal accomplishment is reverse coded and reported as lack of personal accomplishment ${ }^{36}$ so that for all three dimensions higher values indicate greater risk of burnout. We used the most commonly accepted criteria to identify all participants with burnout symptoms-emotional exhaustion score of $\geq 27$ or a depersonalization score of $\geq 10 .^{14}$
Self-identified burnout

The self-identified burnout measure consists of two items: 'I have experienced an episode of burnout-yes or no' and 'Do you still feel you are experiencing burnout?-yes or no.' These items yield three possible response conditions: 'currently burned out,' "formerly burned out' and 'never burned out'. These responses were correlated with the MBI-HSS criteria for burnout. ${ }^{15} 16$ This instrument was originally piloted and tested in a national sample of 2837 perianesthesia nurses. ${ }^{17}$

Veterans RAND 12-item Health Survey

The Veterans RAND 12-item Health Survey (VR-12) was developed from the Veterans RAND 36-item Health Survey, which was developed and modified from the original RAND version of the 36-item Health Survey V.1.0. The VR-12 ${ }^{12} 18$ is a nonproprietary alternative to the 12 -item Short Form Survey ${ }^{17}$ intended to assess physical and mental state. Both of these lowresponse-burden instruments have been successfully used to evaluate health-related quality of life issues and work-related stress in many professions (including medical professionals) and in patients. ${ }^{19-21}$ The VR-12 has been used in large population health surveys by the Veterans Administration and by the Centers for Medicare and Medicaid Services. Physical Composite Score (PCS) and Mental Composite Score (MCS) 2223 are calculated from the responses, with scores ranging from 0 (worst health state) to 100 (best health state). We used the established VR-12 scoring algorithm which was originally designed so that the average score among the population of the USA is 50 with an SD of 10. The VR-12 has age-specific comorbidity associated controls so population values will vary over time. ${ }^{12}$

\section{Social support and personal coping}

The Social Support and Personal Coping (SSPC-25) Survey ${ }^{317}$ is intended to elucidate an individual's coping strategies and social support system. Since it was to be administered in combination with a validated instrument for mental and physical health (the VR-12), we eliminated redundant questions. The remaining 14 questions (the SSPC-14) fall into four natural groupingswork satisfaction, workload and control, professional support and personal support. Each question uses a nine-point scoring system where a higher score represents better coping/support. As in prior work, ${ }^{317}$ we included questions related to relationships (eg, marital status, etc), support from these relationships and personal activities/hobbies (eg, active, distractive or creative). Physical activities were divided into strenuous (eg, running, biking, going to the gym, tennis), moderate (eg, walking, golfing, bowling, dancing) or light/mindful (eg, yoga, meditation, Tai chi) activity. Distractive activities included shopping, reading, going to the theater or movies, traveling, listening to music or watching TV or consuming adult beverages. Creative activities included making music or art or cooking.

\section{Statistical analysis}

Data were analyzed by subspecialty group. Descriptive summaries were calculated for participants' characteristics (age, gender, job title, work area) and constructs from the four study instruments. Counts and percentages were used for categorical variables and the medians and (25th and 75th percentiles) for continuous variables. The Kruskal-Wallis test and the Pearson $\chi^{2}$ test were used, as appropriate, to compare scores between various professional roles. All of the statistical analyses were specified a priori.

To examine the associations between burnout and the risk factors, which included participant age, gender, job title, 
components of the VR-12 and SSPC, the reported substance use and hobbies, ordinary linear regression analysis and the logistic regression analysis were used for MBI subscales and burnout status (emotional exhaustion score $\geq 27$ or depersonalization score $\geq 10$ ), respectively. Effect sizes as well as their $95 \%$ CIs and $\mathrm{p}$ values are reported in the fully adjusted context. The Spearman's correlation coefficient was calculated to compare MBI-HSS and self-identified burnout. In addition, sensitivity, specificity, positive predictive value and negative predictive value and their 95\% CIs were calculated to assess the validity of the self-identified burnout measurement.

All analyses were implemented using R V.3.5.2 (R Foundation for Statistical Computing, Vienna, Austria). To account for multiple comparisons, a significance level of 0.01 was used for statistical inference.

\section{RESULTS}

\section{Demographics}

In all, 1303 participants began the survey, and 118 had missing data; 353 respondents belonged to ASRA, 496 to SPA and 336 to SCA. ASRA and SPA invited approximately 3100 participants of which 1100-1300 opened the emails. SCA invited 3500 and approximately 1550 opened the emails. Based on the number of eligible participants who received the invitations and the number who actually opened the emails, the response rates were $30.5 \%$ for ASRA, $35.6 \%$ for the pediatric, and $21.6 \%$ for the cardiac cohorts. Of the ASRA respondents, $1 / 3$ did not identify as doing either chronic or acute pain. Additionally, this $1 / 3$ was not used in comparisons of burnout. Of those who did reply, approximately $70 \%$ self-identified as primarily doing acute pain (the 'acute' group) and 20\% self-identified as chronic pain providers (the 'chronic' group). A total of 1065 completed surveys were available for analysis (online supplemental table S1).

Age distribution was bimodal (online supplemental table S1), with more participants in the $40-49$ years or $60+$ years and fewer in the 50-59 age range. Compared with the other groups, proportionally more respondents were $60+$ in the acute group $(\mathrm{p}=0.01)$, and more were still in training in the chronic group $(\mathrm{p}=0.03)$. The proportion of females in the pediatric $(49 \%)$ and chronic (41\%) groups was appreciably higher than in the acute or cardiac groups (the overall test $\mathrm{p}<0.001$ ). A larger percentage of participants in the pediatric and cardiac groups worked in an academic setting than did ASRA participants $(p<0.001)$. Thirtyfour per cent of those self-identifying as doing predominantly chronic pain practiced in an office-based setting compared with virtually no office-based practice in the other three groups $(\mathrm{p}<0.001)$.

\section{Maslach Burnout Inventory Human Services Survey}

Burnout symptoms were evident, according to established criteria (see the Methods section) in $43.8 \%$ of combined study participants (table 1). Emotional exhaustion scores were the highest (ie, more burnout) and lack of personal accomplishment scores were the lowest (less) in all groups (figure 1). All subspecialties had lower (better) lack of personal accomplishment scores compared with the normative MBI-HSS data (indicated by an $\mathrm{X}$ on the figures). The acute group was similar to control for emotional exhaustion and depersonalization. The chronic group had significantly higher (worse) emotional exhaustion and depersonalization scores than control, whereas pediatric had lower (better) scores in all three MBI subscales than control. Cardiac had a lower (better) depersonalization score than the control but no significant difference in emotional exhaustion.
Table 1 The impact of subspecialist characteristics stratified by burnout symptoms present or absent measured by MBI-HSS ${ }^{*} \dagger$

\begin{tabular}{|c|c|c|c|}
\hline & Combined & No burnout & Burnout \\
\hline Group sample size & 1288 & 723 & 565 \\
\hline \multicolumn{4}{|l|}{ Age in years, $\mathrm{n}(\%)$} \\
\hline $25-39$ & $125(17)$ & $128(23)$ & $253(20)$ \\
\hline $40-49$ & $147(20)$ & $171(30)$ & $318(25)$ \\
\hline $50-59$ & $190(26)$ & $144(26)$ & $334(26)$ \\
\hline $60+$ & $259(36)$ & $121(21)$ & $380(30)$ \\
\hline Female, $n(\%)$ & $246(34)$ & $206(36)$ & $452(35)$ \\
\hline \multicolumn{4}{|l|}{ Education level, \% } \\
\hline Attending & $620(86)$ & $473(84)$ & $1093(85)$ \\
\hline Resident/fellow & $100(14)$ & $90(16)$ & $190(15)$ \\
\hline \multicolumn{4}{|c|}{$\begin{array}{l}\text { VR-12, median (25th, 75th } \\
\text { percentile) } \ddagger\end{array}$} \\
\hline PCS & $55(48,58)$ & $56(49,58)$ & $54(47,59)$ \\
\hline MCS & $45(35,54)$ & $51(44,56)$ & $35(29,43)$ \\
\hline \multicolumn{4}{|c|}{ SSPC, median (25th, 75th percentile)§ } \\
\hline Work satisfaction & $5(4,6)$ & $5(4,6)$ & $4(3,5)$ \\
\hline Personal support & $4(2,6)$ & $5(3,6)$ & $2(2,5)$ \\
\hline Work control & $2(2,4)$ & $3(2,5)$ & $2(1,3)$ \\
\hline Professional support & $5(4,6)$ & $5(5,6)$ & $4(4,5)$ \\
\hline \multicolumn{4}{|c|}{ Participation in activities, n (\%) } \\
\hline Physical activities & $639(88)$ & $447(79)$ & $1086(84)$ \\
\hline Distractive activities & $557(77)$ & 441 (78) & $998(77)$ \\
\hline Creative activities & $330(46)$ & $201(36)$ & $531(41)$ \\
\hline
\end{tabular}

${ }^{*} P$ values were calculated using the Wilcoxon rank sum test and the Pearson $\chi^{2}$ test as appropriate. Fifteen participants had missing burnout data.

tBurnout symptoms are considered present if emotional exhaustion $\geq 27$ or depersonalization $\geq 10$ per established criteria.

¥PCS and MCS are scored on a range of 0 (worst) to 100 (best) with 50 being the average score of the United States population with a standard deviation of 10. $\S S S P C$ scores range from 0 to 9 where a higher score shows better coping/support. MBI-HSS, Maslach Burnout Inventory-Human Services Survey; MCS, Mental Composite Score; PCS, Physical Composite Score; SSPC, Social Support and Personal Coping; VR-12, Veterans Rand 12-Item Health Survey.

The chronic cohort had worse emotional exhaustion and depersonalization, that is, more burnout, than the acute, pediatric and cardiac $(\mathrm{p}<0.001$, table 2$) .62 .3 \%$ of the Chronic group manifested burnout symptoms, much greater than any of the other groups $(\mathrm{p}<0.001)$.

Burnout was more common among younger physicians (vs older) across all groups (figure 2). Compared with those who were 60 and older, the age category 25-39 was associated with a higher emotional exhaustion score in the chronic group ( $\mathrm{p}=0.003)$. The age category $25-39$ was also associated with increased depersonalization in the chronic, pediatric and cardiac $(p<0.001)$ groups and with increased lack of personal accomplishment in the pediatric and cardiac $(\mathrm{p}<0.001)$ groups. The 40-49 age range was associated with increased lack of personal accomplishment in the pediatric $(\mathrm{p}<0.001)$ and cardiac $(\mathrm{p}<0.001)$ groups. The $50-59$ age group was not different from the $60+$ group in any specialty.

Females in the chronic group were lower in emotional exhaustion. Females in the pediatric and cardiac groups were lower in depersonalization. All other aspects of burnout were no different than in males. For the established criteria for burnout symptoms (emotional exhaustion $\geq 27$ or depersonalization $\geq 10$ ), after adjusting for covariates, the only significant associations were younger members (age $<50$ compared with $60+$ yearsold) of the cardiac group (figure 2) and female members of the pediatric group. 
Average score for MBI-HSS sub scale in subspecialty anesthesiologists
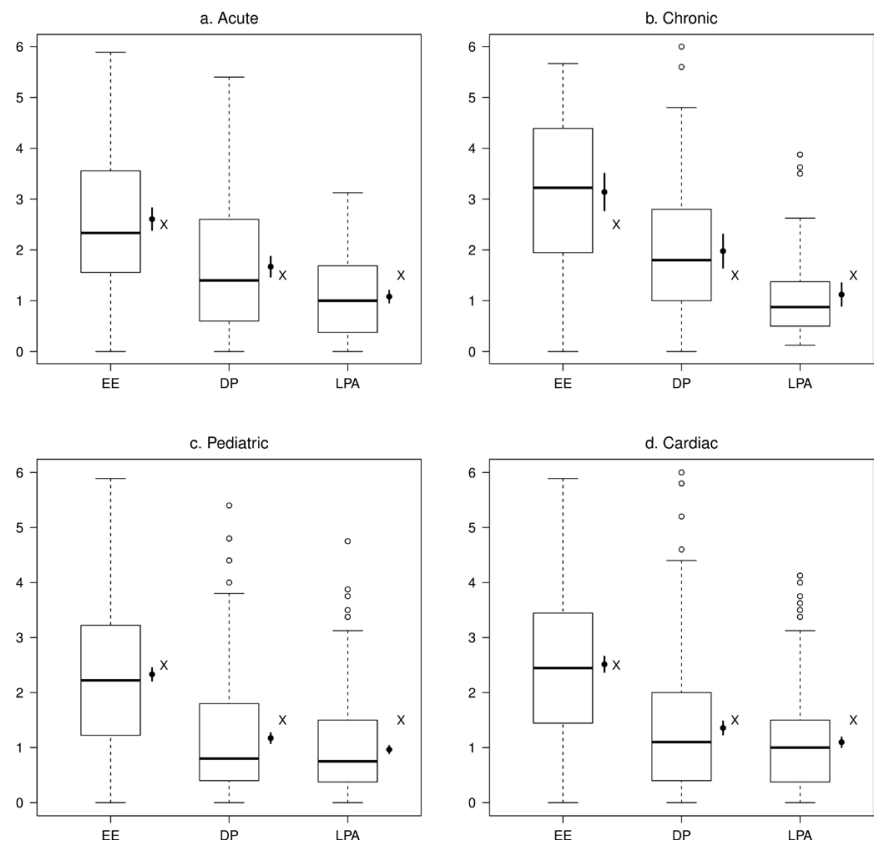

Figure 1 These box-and-whisker plots demonstrate the distribution of average scores for MBI-HSS subscales in subspecialty anesthesiologists- EE (nine questions), DP (five questions) and LPA (eight questions). Answers are given on a seven-point Likert scale (encoded $0-6$ ) so the maximum value for each dimension could be: EE (54), DP (30), LPA (48). The $y$-axis is the average score (total score divided by the number of questions in each respective category) so that all measures are in the same range. Black dots and segments represent mean values and their $95 \% \mathrm{Cls}$. Normative controls are displayed by an ' $X$,' which represents results from 1104 providers-(physicians and nurses $)^{4}$ matched only by profession. higher values predict more risk of burnout. DP, depersonalization; EE, emotional exhaustion; LPA, lack of personal accomplishment; MBI-HSS, Maslach Burnout Inventory Human Services Survey.

\section{MBI-HSS and VR-12}

Each subspecialty had overall PCSs that were better than VR-12 norms, but the MCSs were much worse than VR-12 norms. Mental composite score was inversely associated with all aspects of MBI-HSS in all four specialty groups $(\mathrm{p}<0.001$ except depersonalization in chronic). PCS was inversely associated with all three dimensions of MBI-HSS in the cardiac group $(\mathrm{p}<0.001)$ but not with any dimension of MBI-HSS in the chronic group. The incidence of burnout symptoms using MBI-HSS criteria (see the Methods section) was adversely affected by lower MCS and PCS in both the Cardiac and pediatric groups (figure 2). In contrast, burnout was not associated either with mental or PCS in the chronic group and with only MCS in the acute group.

\section{Self-identified burnout}

There was no significant effect of age or gender on self-identified burnout. There was more 'currently burned out' and less 'never burned out' in the chronic compared with all three other groups $(p=0.02$, table 2$)$. There were no statistically significant differences in self-identified burnout between the acute, pediatric and cardiac groups.

\section{Mental and physical health metrics}

MCS and PCS were similar in all subspecialty groups. PCS was better than normal and MCS was worse. Separating the specialties by self-identified burnout reveals other data. MCS was lowest in all subgroups in those self-reporting current burnout, but this effect was not seen with PPCS. Pediatric and Cardiac 'Never burned out' were the only subgroups with MCS at or near norm (table 1). With the exception of the chronic group, MCS was never as good in the current and former subgroups as in the never subgroup.

\section{MBI-HSS compared with Self-Identified burnout}

Self-identified burnout was associated with all MBI-HSS. Those reporting being currently burned out were more likely to have one or more high MBI-HSS subscale scores (online supplemental table S2), and all three MBI subscales (emotional exhaustion, depersonalization and lack of personal accomplishment) had worse average scores in 'formerly burned out' and 'never burned out' respondents than in those reporting 'current burn out'.

To more precisely assess the relationship of self-identified burnout with MBI-HSS dimensions and with burnout symptoms, we calculated the Spearman's correlation coefficient (R), sensitivity, specificity, positive predictive value, negative predictive value and their 95\% CIs (online supplemental table S3). Self-identified burnout was most strongly associated with emotional exhaustion and least strongly with lack of personal accomplishment. The overall positive predictive value of the self-identification measure (ie, reported burnout when burnout

Table 2 MBI-HSS scores and burnout parameters by subspecialty group*

\begin{tabular}{|c|c|c|c|c|c|}
\hline & All respondents & Acute & Chronic & Pediatric & Cardiac \\
\hline Sample size, $\mathrm{n}$ & 1303 & 164 & 69 & 496 & 336 \\
\hline Burnout identified by MBI-HSS ( $E E \geq 27$ or $D P \geq 10), n(\%) \dagger \ddagger$ & $565(43)$ & $74(45)$ & $43(62)$ & $149(30)$ & $189(56)$ \\
\hline EE score, median (25th, 75th percentile) & $22(13,31)$ & $21(14,32)$ & $29(18,39)$ & $20(11,29)$ & $22(13,31)$ \\
\hline DP score, median (25th, 75th percentile) & $6(2,10)$ & $7(3,13)$ & $9(5,14)$ & $4(2,9)$ & $6(2,10)$ \\
\hline LPA score, median (25th, 75th percentile) & $7(3,12)$ & $8(3,14)$ & $7(4,11)$ & $6(3,12)$ & $8(3,12)$ \\
\hline \multicolumn{6}{|l|}{ Self-identified burnout, $\mathrm{n}(\%) \S$} \\
\hline Current & $303(23)$ & $33(20)$ & $26(38)$ & $100(20)$ & $82(24)$ \\
\hline Former & $317(24)$ & $39(24)$ & $18(26)$ & $129(26)$ & $72(21)$ \\
\hline Never & $670(51)$ & $92(56)$ & $24(35)$ & $264(53)$ & $182(54)$ \\
\hline
\end{tabular}

*P values were calculated using either the.

†Kruskal-Wallis test.

$\ddagger$ Pearson $\chi^{2}$ test.

§MBI-HSS evaluates EE (nine questions), DP (five questions) and LPA (eight questions). Answers are given on a seven-point Likert scale (encoded 0-6) so the maximum value for each dimension could be: EE (54), DP (30), LPA (48). Higher values predict more risk of burnout.

DP, Depersonalization; EE, Emotional Exhaustion; LPA, Lack of Personal Accomplishment; MBI-HSS, Maslach Burnout Inventory-Human Services Survey 

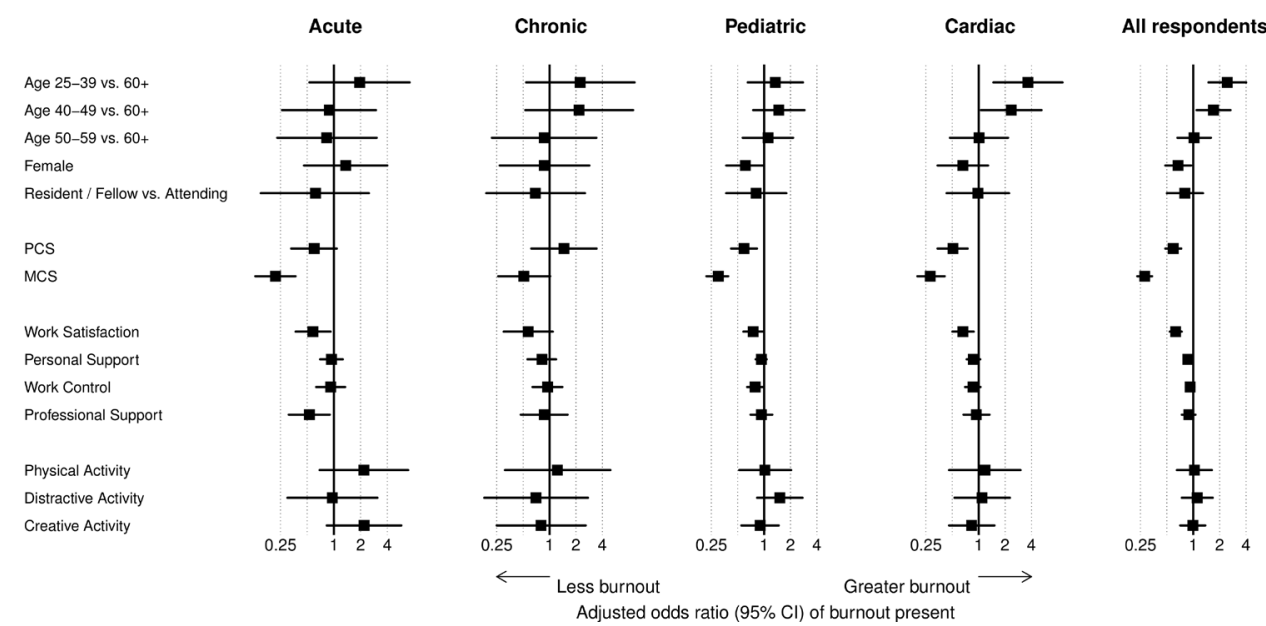

Figure 2 This forest plot demonstrates the impact of easily measured factors on the risk of burnout as determined by MBI-HSS. Criteria for burnout are emotional exhaustion $\geq 27$ or depersonalization $\geq 10$. Covariates adjusted in the model include age, gender, education, substance use, SSPC, VR-12 and activities data. MBI-HSS, Maslach Burnout Inventory Human Services Survey; MCS, Mental Composite Score; PCS, Physical Composite Score; SSPC, social support and personal coping; VR-12, Veterans Rand 12-Item Health Survey.

criteria were met by MBI-HSS standards) was at least 0.93 while the negative predictive value (when burnout was absent) was at least 0.43 .

\section{Social support and personal coping}

Work satisfaction and personal support were significantly associated with burnout present or absent. However, when examining the effect in each subspecialty, only work satisfaction was inversely associated with burnout present in the cardiac group (figure 2). Better work satisfaction was associated with reduced emotional exhaustion in all subspecialties with the exception of the Chronic group and with reduced depersonalization in the pediatric group. Emotional exhaustion seemed to be the most closely associated with work-related SSPC questions, with significant results also for work control (pediatric and cardiac) and for personal support (pediatric only). Professional support was not strongly associated with any MBI-HSS measure. With regard to self-identified burnout, participants who reported being currently burned out had lower SSPC scores than did those who reported being formerly or never burned out.

\section{DISCUSSION}

We evaluated burnout risk in 1303 subspecialty anesthesiologists. Of these, 1065 had complete data and could be categorized into four subspecialty groups-acute or chronic pain, pediatric and cardiac. We had three research questions: (1) does burnout risk as measured by MBI-HSS differ among subspecialty anesthesiologists; (2) does a simple self-reported measure agree with the results of MBI-HSS and (3) do other factors associated with burnout differ between these groups.

Regarding question \#1: the chronic pain group had much higher burnout, as measured by MBI-HSS, than the other three groups, and the latter scored better than comparable MBI-HSS norms in emotional exhaustion and depersonalization. Regarding \#2; the chronic pain group had the highest percentage of those self-identifying as 'currently burned out' and the pediatric cohort had the lowest. Regarding \#3: each subspecialty scored better than Veteran's RAND-12 controls on physical health measurements but were below normal control values for mental health, particularly in the group reported being currently burned out. Social support and personal coping measures are most closely associated with emotional exhaustion and were lower in the Currently burned out participants.

Despite increased attention to the problem of physician burnout in the past 5 years, the proportion of physicians who meet established criteria remains high and is more common than comparable US non-physician employees. ${ }^{24}$ The consequences of physician burnout were recently highlighted by a National Academy of Medicine report, Taking Action Against Clinician Burnout: A Systems Approach to Professional WellBeing. Burned out physicians deliver care that is less safe and less patient centered. ${ }^{25}$ Burned out resident physicians may be more predisposed to occupational injuries. ${ }^{26}$ Further, burned out physicians are more likely to reduce their work hours, leave their current job, and even leave the specialty. ${ }^{25}$ In a 2017 study, $20 \%$ of physicians reported an intent to reduce their workload in the near future and another $2 \%$ intended to leave medicine altogether. ${ }^{27}$ Finally, burned out physicians are more likely to be depressed and even suicidal. ${ }^{28}$ In a 2008 study of surgeons, ${ }^{29} 40 \%$ of participants were likely burned out and $30 \%$ screened positive for depression. Unfortunately, due to stigma and job risk, 40\% of physicians with depression would be reluctant to seek medical help for 'a mental health condition because of concerns about repercussions to their medical licensure'. ${ }^{27}$ Whether through attrition or impairment, loss of physicians due to burnout could pose serious challenges for the nation's healthcare workforce in the near future, not to mention incalculable societal costs. ${ }^{25}$

The MBI-HSS, the 'gold standard' for evaluating burnout, involves time and expense to administer and to answer the 22 questions. A simple, inexpensive, robust screening tool could help identify at-risk individuals. Single-item measures of depersonalization and emotional exhaustion (one question for each) ${ }^{15} 16$ have been used to identify burnout, but are proprietary and may not be sufficiently sensitive or specific compared with the full MBI-HSS. The self-identified burnout questions depend on the respondent's a priori understanding of what constitutes 'burnout.' Self-identified burnout psychometrics correlated highly with, and had excellent positive predictive value compared with MBI-HSS. Further studies will be required to ascertain whether the temporal component of self-identified burnout (ie, currently, formerly or never burned out) has additional value. We previously evaluated self-identified burnout in 
2837 postanesthesia care unit (PACU) nurses. ${ }^{17}$ In that study, fewer nurses were currently burned out than physicians, and half were never burned out, but formerly burned out nurses exceeded physicians by at least $10 \%$.

Those 'currently burned out' have lower MBI-HSS values, and those 'formerly burned out' do not unanimously return to the never burned-out levels. MCSs also suffer more in currently burned out respondents. Similar findings were seen in PACU nurses. ${ }^{17}$ This suggests not only that burnout adversely affects mental health, but also that individuals, once burned out, may not return to 'normal' for quite some time. On the other hand, the failure to return to 'baseline' could be a positive effect since, in recovering from burnout, the individual has created selfprotective personal boundaries that did not previously exist.

This study has several limitations. As with any survey, the potential exists for both selection and response bias. With the cooperation of three major anesthesiology subspecialty societies, we cold-contacted all their members. Using standard methods, our response rate of $22 \%-36 \%$ by subspecialty society is above average and generally deemed acceptable for this methodology. Our overall prevalence of burnout symptoms $(\sim 43 \%)$ is similar to the results found, using similar methods, by Shanafelt et al in their 2017 large survey of a cross-section of US physicians (43.9\%) and slightly higher than that group's 2019 results from anesthesiologist respondents (41\%). ${ }^{24}$ There is inherent bias, because neither a truly random sample nor total participation is possible. Some people may have an interest in the topic and be more likely to participate, while others may believe the topic does not apply to them or under-participate for other reasons, including being too burned out. ${ }^{611}$ Given that the chronic group overall had less favorable results, it would have been desirable to have a larger sample and participation rate from that group. Lack of honest answering is another potential weakness of the study, but we believe, based on prior experience ${ }^{6}$ that people answer these types of questions honestly if they have assurances of anonymity. Alternative approaches such as the double survey method $^{30}$ may be useful in future studies to further mitigate and quantify the risk of response bias.

As described in detail in the National Academy of Medicine report, burnout occurs when the stresses and demands of the job persistently exceed the accessible organizational and individual resources for coping with those demands. ${ }^{24}$ For the vast majority of burned out clinicians, system (ie, organizational and external) contributory factors are much more important than individual states and traits. While the present study provides only limited explanatory data, it is reasonable to postulate that the practice model of the chronic pain anesthesiologists, a model that differs substantially (ie, clinic-based care, greater electronic health record use, surgical care, more need for billing and documentation, etc) from OR-based anesthesiologists, may contribute to their higher incidence of burnout and associated findings in this study. Factors contributing to decreased burnout in pediatric anesthesiologists may include selection bias or perhaps cultural differences in pediatric versus adult perioperative care. Further prospective and probably longitudinal studies are required to elucidate what, if any, aspects of the subspecialty anesthesiologists' jobs are contributing to burnout.

Acute pain, chronic pain, pediatric and cardiac subspecialists appear to have lower at-risk numbers than anesthesiologists-at-large, but more than $40 \%$ on average met the criteria for burnout symptoms, and nearly $25 \%$ self-identified as being currently burned out. With lower than normal MCSs and strong association with burnout, the risk for development of depression or other related mental disorders is significant. Healthcare organizations and professional societies need to be vigilant about the well-being of their members. Our findings support a growing body of literature advocating for more national investment in creating, studying, and implementing systemoriented interventions to prevent and treat clinician burnout.

In conclusion, we evaluated burnout risk in 1303 members of the ASRA, the Society for Pediatric Anesthesia and the SCA. The chronic pain group had the highest amount of burnout measured by MBI-HSS and the highest proportion who self-identified as currently burned out; the pediatric group had the lowest. All groups had below-normal mental health scores, particularly in those who were currently burned out. Our self-identified burnout instrument compared favorably to previously used single question results based on MBI-HSS. ${ }^{1516}$ While the self-identified burnout instrument needs further validation, it may be a useful adjunct as a screening tool or as part of other surveys where low expense and fewer questions would be desirable.

\section{Author affiliations}

${ }^{1}$ Anesthesiology, Vanderbilt University Medical Center, Nashville, Tennessee, USA ${ }^{2}$ Nursing Research, Vanderbilt Nursing, Vanderbilt University Medical Center, Nashville, Tennessee, USA

${ }^{3}$ Anesthesiology, University at Buffalo Jacobs School of Medicine and Biomedical Sciences, Buffalo, New York, USA

${ }^{4}$ Roswell Park Cancer Institute, Buffalo, New York, USA

${ }^{5}$ Biostatistics, Vanderbilt University Medical Center, Nashville, Tennessee, USA ${ }^{6}$ Anesthesiology, Biomedical Informatics, Medical Education, Vanderbilt University Medical Center, Nashville, Tennessee, USA

${ }^{7}$ Center for Research and Innovation in Systems Safety, Vanderbilt University Medical Center, Nashville, Tennessee, USA

\section{Twitter Steve A Hyman @VUMC_Anes}

Acknowledgements The American Society of Regional Anesthesia and Pain Medicine (ASRA) was involved in conceiving the study, provided guidance on study planning, and facilitated data collection. Some of these data were presented in poster form at the ASRA 2017 Annual Meeting. The VR-12 was developed at RAND as part of the Medical Outcomes Study.

Contributors SAH: literature search, study design, data collection, data analysis and interpretation, writing and editing manuscript. EBC: study design, data collection, data interpretation, writing and editing manuscript. ODL-C: study design, writing and editing manuscript. MSS: study design, data analysis and interpretation, figures, writing and editing manuscript. YS: data analysis and interpretation, figures, writing and editing manuscript. MBW: study design, data analysis and interpretation, writing and editing manuscript.

Funding Access to study participants was provided by the American Society of Regional Anesthesia and Pain Medicine (ASRA), the Society for Pediatric Anesthesia (SPA), and the Society of Cardiovascular Anesthesiologists (SCA). Biostatistical and manuscript preparation support was provided by the Department of Anesthesiology and the Center for Research and Innovation in Systems Safety (CRISS).

Competing interests None declared.

Patient consent for publication Not required.

Provenance and peer review Not commissioned; externally peer reviewed.

Data availability statement Data are available on reasonable request. Data are available on reasonable request. Data are deidentified participant data. Data may be available from SAH (ORCID id: 0000-0002-3888-3924), the corresponding author, on request and execution of a data use agreement.

ORCID iD

Steve A Hyman http://orcid.org/0000-0002-3888-3924

\section{REFERENCES}

1 Freudenberger HJ. The staff burnout syndrome in alternative institutions. Psychol Psychother 1975;12:73-82.

2 Freudenberger HJ. Burnout: occupational hazard of the child care worker. Child Care Q 1977;6:90-9.

3 Hyman SA, Michaels DR, Berry JM, et al. Risk of burnout in perioperative clinicians: a survey study and literature review. Anesthesiology 2011;114:194-204.

4 Maslach C. MBI Manual. In: Jackson S, ed. ed. 3rd edn. Mountain View, CA: CPP, Inc, 1996.

5 Sun H, Warner DO, Macario A, et al. Repeated cross-sectional surveys of burnout, distress, and depression among anesthesiology residents and first-year graduates. Anesthesiology 2019;131:668-77. 
6 Hyman SA, Shotwell MS, Michaels DR, et al. A survey evaluating burnout, health status, depression, reported alcohol and substance use, and social support of Anesthesiologists. Anesth Analg 2017;125:2009-18.

7 Committee on Systems Approaches to Improve Patient Care by Supporting Clinician Well-Being, National Academies of Sciences, Engineering, and Medicine. Taking action against clinician burnout : a systems approach to professional well-being In. Washington DC: The National Academies Press, 2019: 81-126.

8 Back AL, Steinhauser KE, Kamal AH, et al. Building resilience for palliative care clinicians: an approach to burnout prevention based on individual skills and workplace factors. J Pain Symptom Manage 2016;52:284-91.

9 Harrison KL, Dzeng E, Ritchie CS, et al. Addressing palliative care clinician burnout in organizations: a workforce necessity, an ethical imperative. J Pain Symptom Manage 2017;53:1091-6.

10 Harris PA, Taylor R, Thielke R, et al. Research electronic data capture (REDCap)--a metadata-driven methodology and workflow process for providing translational research informatics support. J Biomed Inform 2009;42:377-81.

11 Dillman DA, Smyth JD, Christian LM. Internet, mail, and Mixed-mode surveys: the tailored design method. 3rd edn. Hoboken, N.J: Wiley \& Sons, 2009.

12 Selim AJ, Rogers W, Fleishman JA, et al. Updated U.S. population standard for the Veterans Rand 12-Item health survey (VR-12). Qual Life Res 2009;18:43-52.

13 Maslach C, Schaufeli WB, Leiter MP. Job burnout. Annu Rev Psychol 2001;52:397-422.

14 Rotenstein LS, Torre M, Ramos MA, et al. Prevalence of burnout among physicians: a systematic review. JAMA 2018;320:1131-50.

15 West CP, Dyrbye LN, Sloan JA, et al. Single item measures of emotional exhaustion and depersonalization are useful for assessing burnout in medical professionals. J Gen Intern Med 2009;24:1318-21.

16 West CP, Dyrbye LN, Satele DV, et al. Concurrent validity of single-item measures of emotional exhaustion and depersonalization in burnout assessment. J Gen Intern Med 2012;27:1445-52.

17 Card EB, Hyman SA, Wells N, et al. Burnout and Resiliency in Perianesthesia nurses: findings and recommendations from a national study of members of the American Society of PeriAnesthesia nurses. J Perianesth Nurs 2019;34:1130-45.

18 Kazis LE, Selim A, Rogers W, et al. Dissemination of methods and results from the Veterans health study: final comments and implications for future monitoring strategies within and outside the Veterans healthcare system. J Ambul Care Manage 2006;29:310-9.

19 Kudielka BM, Hanebuth D, von Känel R, et al. Health-Related quality of life measured by the SF12 in working populations: associations with psychosocial work characteristics. J Occup Health Psychol 2005;10:429-40.

20 Voltmer E, Schwappach DLB, Frank E, et al. Work-Related behavior and experience patterns and predictors of mental health in German physicians in medical practice. Fam Med 2010;42:433-9.

21 Walt JG, Rendas-Baum R, Kosinski M, et al. Psychometric evaluation of the glaucoma symptom identifier. J Glaucoma 2011;20:148-59.

22 Ware JE, Kosinski M, Keller SD. SF-12: how to score the SF-12 physical and mental health summary scales. Boston Massachusetts: The Health Institute, New England Medical Center, 1998.

23 Ware J, Kosinski M, Keller SD. A 12-Item short-form health survey: construction of scales and preliminary tests of reliability and validity. Med Care 1996;34:220-33.

24 Shanafelt TD, West CP, Sinsky C, et al. Changes in burnout and satisfaction with worklife integration in physicians and the general us working population between 2011 and 2017. Mayo Clin Proc 2019;94:1681-94.

25 Committee on Systems Approaches to Improve Patient Care by Supporting Clinician Well-Being, National Academies of Sciences, Engineering, and Medicine. Taking action against clinician burnout : a systems approach to professional well-being. Washington DC: The National Academies Press, 2019: 63-80.

26 West CP, Tan AD, Shanafelt TD. Association of resident fatigue and distress with occupational blood and body fluid exposures and motor vehicle incidents. Mayo Clin Proc 2012;87:1138-44.

27 Dyrbye LN, West CP, Sinsky CA, et al. Medical licensure questions and physician reluctance to seek care for mental health conditions. Mayo Clin Proc 2017;92:1486-93.

28 Shanafelt TD, Balch CM, Dyrbye L, et al. Special report: suicidal ideation among American surgeons. Arch Surg 2011;146:54-62.

29 Shanafelt TD, Balch CM, Bechamps GJ, et al. Burnout and career satisfaction among American surgeons. Ann Surg 2009;250:107-15.

30 Johnson TP, Wislar JS. Response rates and nonresponse errors in surveys. JAMA 2012;307:1805-6. 